\title{
NEUROCIENCIAS MOLECULARES EN PSICOLOGÍA, PSICOANÁLISIS E INCONSCIENTE
}

\section{MOLECULAR NEUROSCIENCE IN PSYCHOLOGY, PSYCHOANALYSIS AND UNCONSCIOUS}

Ana Maria Montero Doig

\section{Resumen}

El presente artículo tiene como finalidad mostrar algunas correlaciones entre estudios recientes en Neurociencias Moleculares en Psicología y fenómenos clínicos descritos por los Psicoanalistas, así como el inconsciente; asimismo, se comentan algunas investigaciones correspondientes respecto a las redes neuronales convergentes y divergentes, sus conexiones, el rol de las impresiones/ asociativas en la cadena del dato y sus bases de conducción, rutas, circuitos y archivos mnémicos, describiendo como la percepción inconsciente se fija en diversas regiones. Así mismo se toma en cuenta las huellas significativas afectivas en los periodos críticos del desarrollo psicológico antes y después de la edad responsable, las influencias de las instituciones, el rol de la educación y el libre albedrío sensorial-perceptivo.

Palabras clave: Neurociencias Moleculares; Psicoanálisis Moderno; Percepción inconsciente (Sueños, Actos fallidos y el chiste); Redes Neuronales.

\section{Abstract}

The purpose of this article is to show some correlations between recent studies in Molecular Neurosciences in Psychology and clinical phenomena described by Psychoanalysts, as well as the unconscious; likewise, some corresponding research is commented on convergent and divergent neural networks, their 
connections, the role of impressions / associations in the data chain and their bases of conduction, routes, circuits and mnemonic files, describing how unconscious perception is fixed in different regions. Likewise, significant affective traces are taken into account in critical periods of psychological development before and after responsible age, the influences of institutions, the role of education and the sensorial-perceptual free will.

Key words: Molecular Neurosciences; Modern Psychoanalysis; Unconscious Perception (Dreams, Failed Acts and the joke); Neural Networks.

\section{Introducción}

El presente artículo consta de cinco partes. En la Introducción se presenta una idea sobre los fotones en el cerebro, dado que una vez que este recibe la luz produce sustancias Neuropéptidos y NT neurotransmisores biofotónicos: Sustancias químicas como: Dopamina, Serotonina Noradrenalina, Adrenalina; todas sustancias moleculares de impacto en la Psicología Clínica, específicamente en el núcleo de las neuronas ARN, qué le dice qué hacer al núcleo $\mathrm{ADN}$ a través de la proteína Turbulina en la estructura de los cito esqueletos plasmáticos y esta es la memoria celular relacionada con el inconsciente porque son sistemas de defensas (MDI -MDE), que se originan cuando las neuronas sirven al instinto de conservación y reaccionan con un movimiento (dopamina) con miedos o cólera al riesgo (Adrenalina - Noradrenalina) y ante la frustración o falta de placer con tristeza (serotonina) y que sirven al cuerpo humano para reaccionar ante determinadas circunstancias, la pérdida de su energía fotonica causa déficit o el excesos en el uso termo dinámico de la energía celular, del órgano o del sistema del cuerpo fi- sico; donde el NT neurotransmisores o NP neuropéptidos reaccionan como biofotónes físicos-químicos en las sinapsis, que provocan que estructuras del SNV sistema nervioso vegetativo, por ejemplo, haga que el corazón lata muy rápido o muy lento y se observa individuos eufóricos o con hundimientos afectivos, expresiones observadas en el flujo de las redes neuronales asociativas RNAs. Por ejemplo caso de red RNAs entre Sensación y Emoción negativa donde la glándula pineal adapta su ciclo al de la Tierra, vemos que las 12 horas del día en la Tierra son las 12 horas de vigilia para la glándula pineal, la cual está constantemente en armonía con el ciclo horario terrestre, el déficit de un NT (dopamina, serotonina, adrenalina), por eso es posible observar a las neuronas dopaminérgicas que al liberar dopamina a su vez transmiten el mensaje de sensación placentera y euforia cualquiera que sea es una consecuencia perdida de energía o de hundimiento emocional donde la glándula pineal habitualmente reacciona causando sueño o fatiga física o sensorial (a su vez decae el nivel de atención del locus cereleus en la FRA, formación reticular ascendente). En la segunda parte, se exponen 
consciencias entre el psicoanálisis y las neurociencias, las convergencias de las redes neuronales de la base material del inconsciente en el cerebro. En la tercera parte, están realizadas mis investigaciones durante más de una década en neurociencias moleculares en psicología.En la cuarta parte, están realizadas las presentaciones de redes neuronales por convergencia y evidencias científicas de la memoria de la célula en ARN y ADN. Finalmente, conclusiones.

Recordando juntos, Dalmasio en el Congreso de la Asociación Psicoanalitica Internacional XLIII, celebrado en Nueva Orleáns en el 2004, es especialmente conocido por su libro, el más popular de todos, traducido a múltiples idiomas, El error de Descartes (1994). Además, antes era insólito pensar que podrian exponerse tesis neurocientíficas ante psicoanalistas de todos lados en una asamblea general. Por otra parte, hechos como que un neurólogo presentase exposiciones sobre tópicos como "la arquitectura emocional del miedo y el placer", ejemplifica las actuales correlaciones entre las neurociencias y el psicoanálisis. Continuando con estas, hace más de una década se editó una revista con el nombre de NeuroPsychoanalysis. Asimismo, las publicaciones de libros respecto a convergencias entre estas disciplinas van en aumento, así como las conferencias respecto a esta correlación.

Sigmund Freud, en 1895, en su trabajo de "una psicología para neurólogos":
Intentó adaptar lo que había comenzado a descubrir sobre el funcionamiento inconsciente de la mente a las rudimentarias nociones de neurociencia de aquel entonces. Freud, consciente de la escasez de conocimientos neurofisiológicos que pudieran dar cuenta de sus tesis psicológicas, decidió no publicar dicho Proyecto. Éste no vio la luz hasta 1950, más de una década después de su muerte (Freud, 1895).

Más adelante, en 1914, Freud (1973), narro: Tendremos que recordar que todas nuestras ideas provisorias psicológicas habrán de ser adscritas alguna vez a substratos orgánicos; Posteriormente, luego de seis años, haciendo alusión a los déficits de sus constructos, narraria: "desaparecerian con seguridad si en lugar de los términos psicológicos pudiéramos emplear los fisiológicos o los químicos" (Freud, 1973). Desafortunadamente, Freud no está vivo para ver cómo luego de un siglo, las convergencias entre ambas disciplinas se van tornando más significativas.

Con el pasar del tiempo, el proyecto del cerebro azul sumado a otros datos respecto a la dinámica del $\mathrm{SNC}$, la neurología se dará cuenta de muchas de las verdades del inconsciente que postula Freud, y esto gracias a las correlaciones entre ambas disciplinas.

Morton Reise (1984), formuló una interrogante hace un par de décadas: “¿Tienen algo que decirse el psicoanálisis y la neurobiología?”. La respuesta en la actualidad es una afirmación por 
categorias, ya que al respecto, Regina Pally (1997), ha indicado: "El reconocimiento de la enorme dependencia de la experiencia adquirida (en la infancia) que tiene el desarrollo cerebral constituye un ejemplo patente de cómo pueden integrarse la neurociencia y el psicoanálisis", Aunado a esto, el Nobel de neurofisiología, Eric Kandel (1983), redactó: "El énfasis puesto por el psicoanálisis en la estructura del psiquismo y en sus representaciones internas ha constituido una de las fuentes de la psicología experimental y cognitiva moderna". El funcionamiento de las redes neuronales da como resultado la vida psíquica, sea la normal o la patológica; por esto, la mente tiene su origen en el cerebro, a su vez, esta víscera, relacionada con los estímulos externos, sumado a la experiencia subjetiva, modifica los procesos estructurales del cerebro. Es así que tenemos la biología que subyace a la función, pero la función modifica a la víscera, y con más ahínco en la infancia.

Hay un fenómeno interesante descrito por Jean Piaget en el año 1950, que alude a una relación del inconsciente con las neurociencias: se trata del fenómeno de la denominada constancia objetal, que consiste en la existencia de la representación mental de una persona $u$ objeto en la ausencia de éste, capacidad que el ser humano adquiere normalmente a lo largo del segundo año de vida. Gracias a este fenómeno, la relación madre-hijo, se solidifica en tiempo y espacio, a pesar de situaciones estresantes que pue- dan poner en riesgo este vínculo. Ahora, este fenómeno descrito hace más de medio siglo, tiene correlación con los descubrimientos de la neurobiologia, pues hoy en día es bien sabido que luego de que la mielinización del SNC se completa, las neuronas del córtex premotor codifican estímulos visuales y permanecen activadas aun cuando no estén presentes los objetos en cuestión o no resulten visibles, como en la oscuridad. Es así que esta permanencia explica la disponibilidad de las impresiones sensoriales para las evocaciones asociativas en ausencia de los estímulos originales. El hecho de que la imaginación por sí misma active las neuronas del córtex correspondiente a la percepción directa por los sentidos, refuerza la tesis de la efectividad de la actividad asociativa, proporcionando una constatación física de algo que los psicoanalistas ya conocían empíricamente.

\section{Circuitos nerviosos y las imáge- nes visuales}

Existen circuitos lineales E-O-R simples y circuitos de redes neuronales asociativas RNAs, más complejas en paralelo sobre lo que aprendemos en el trascurso de la vida, siendo las recepciones de los estímulos visuales y auditivos los que son más rememorados más inconscientemente en el silencio. Esas percepciones tienen una singularidad afectiva y pueden causar o no causar una interacción de la Red Sensorial (SNP) de tipo visual con otros centros del cerebro a través 
de las impresiones Fotónicas, evidentemente en la estructura de los ojos, cuando se agota un pigmento del color de las células llamadas conos, se producen complementarios, llevan las señales. Lo mismo pasa con los pigmentos purpura de los bastones y al lóbulo occipital por eso cada estructura desarrolla funciones psíquicas sutiles tanto en la glándula Pineal (vigiliasueño) como de asociaciones o llamados pensamientos mágico-primitivos o pensamientos racionales lucidos, que acompañan de reacciones motoras, frente a riesgos instintivos de conservación de la especie.

Se ha observado que nuestras posibilidades de comprensión y respuestas adecuadas se ven incrementadas cuando imaginamos un objeto antes de detectarlo con los sentidos, o una situación antes de que ocurra, aunado a esto, hay que añadir los elementos idiosincrásicos a la apercepción de nuestra cosmovisión. Así es como tenemos por cauce natural la impresión subjetiva del mundo tal y como es en sí, sin mencionar que lo fragmentado de nuestra percepción se ve teñida de manera sustancial por las motivaciones, los estados emocionales y las experiencias del pasado. En otros términos, es verosímil afirmar que la percepción es pura. Y esto es corroborado por experimentos de laboratorio y experiencias clinicas que han comprobado que la inmaculada percepción es ilusoria. Pero tal es su importancia que en el abordaje psicoanalitico se hace énfasis en que el analizado desarrolle un nivel máximo de examen de la realidad con un minimo margen de distorsión.

\section{Los recuerdos especificos del in- consciente}

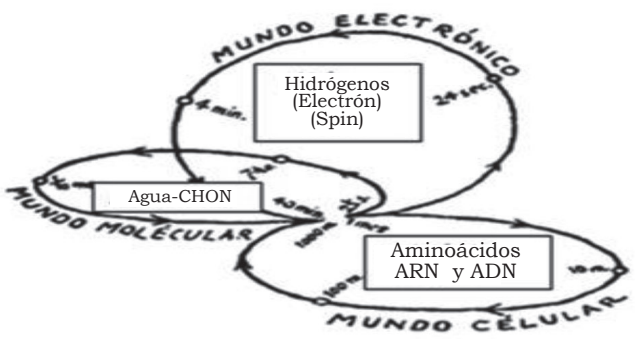

Recorrido de recuerdos específicos en el ARN inconsciente de la célula.

Montero Doig, Ana María 2018

E1 recuerdo es un proceso de evocación fotónica del mundo eléctrico, molecular y de los aminoácidos del ARN ADN. El modelo de la "codificación específica" (Tulving y Thompson, 1973, p.352). Post mis investigaciones señalo que el valor del inconsciente de la célula en su nivel sub celular iónico existen huellas de impresiones fotónicas con cadenas de asociaciones de evocación, entre los procesos de reconocimiento y evocación, que forman redes de convergencia del recuerdo dado conjuntos de imágenes de hechos o circunstancias del pasado registrado que quedan en la mente como recuerdos de si. Todo este proceso está formado por parte en la memoria sensorial (MS). (MONTERO D, 2018)

En todas las etapas evolutivas existen receptores sensoriales con mecanismos eléctricos $(\mathrm{mV})$, moleculares químicos diatómicos y poli atómicos neurotransmisores/neuropéptidos 
(NT) (Np) en el cuerpo físico, grabados fotónicamente a nivel subcelular; sea del recién nacido, infantes, jóvenes, adultos y ancianos, es así que los primeros meses de la vida tenemos base de datos (BD) con densidad, frecuencia y velocidad expresados en el (PA) potencial de acción del estímulo y de reposo (PR), todo un mecanismo del electrón o fotón, mundo del electrón y molecular químico $\mathrm{CHON}$ con significados y significantes que no recordamos hasta los 3 años, porque los recuerdos fueron solo almacenados automáticamente (como fotografias) en las células del cuerpo donde las moléculas de las emociones tuvieron mayor impacto y con el rol metabólico predominante del hipotálamo centro de necesidades básicas y que coordina la central de evocación del hipocampo proceso de evocación del recuerdo, en nuestras redes neuronales mnémicas (MONTERO D, 2018).

Las neurociencias moleculares en psicología afirman las teorias de Melanie Klein, que atribuyen al neonato cogniciones complejas, afirmando que las funciones mnémicas de los ganglios basales (nuestro cerebro motorinstintivo) y de la amígdala (cerebro emocional) son compartidas por los mamíferos inferiores, ya que en el recuento filogenético, los neonatos ya la tienen bien desarrolladas.

La memoria motora operativa de los automatismos está registrada en el cuerpo estriado, mientras que el aprendizaje de las respuestas emocionales se da en la amígdala límbica. Gracias a estas redes de convergencia en el SNC se origina la memoria implícita no verbal que está desarrollada en los infantes a través de sobresaltos, síntomas somáticos, sensaciones placenteras, temores primarios y la interacción con la madre (vínculos de protección o de abandono/postergación), que no son evocados por falta de una memoria explícita (o declarativa) que ejerce la instrucción o educación escolástica.

A partir del año y medio, la capacidad verbal del neonato coincide con la maduración de la estructura del hipocampo; esto genera la hipótesis de que una adecuada interacción vocal con el niño va a generar una facilidad en el proceso de los recuerdos infantiles por la memoria explícita, por ende, este tendrá acceso a la rememoración consciente.

Hasta el desarrollo de la conciencia moral, los siete años, las redes continúan su mielinización, así como las redes que conectan el sistema límbico y el córtex, que, como consecuencia, arroja una integración progresiva de la actividad cognitiva con lo afectivo (peligroso punto de la experiencia temprana infantil porque las emociones pueden equivocar el pensamiento $\mathrm{y}$ viceversa).

En las neurosis postraumáticas de pacientes infantes y adultos, puede apreciarse que hay una disociación entre la implícita, que pertenece a la amígdala, explícita, que pertenece al hipocampo.

Las relaciones objétales según Melanie Klein, demuestran que las estructuras psíquicas se originan al 
internalizar (interiorizar) las experiencias de relación con los objetos que son recuerdos significativos, donde los hechos de abandonos o postergaciones hacen que el paciente germine patologías, por consiguiente, sufra crisis de miedos o angustia somatizada y otros sintomas físico-químicos en el torrente sanguíneo como dice las neurociencias moleculares (Montero 2016), pues estas están teñidas con inyecciones de adrenalina sin recuerdos explícitos que han de ir relacionados (carga inconsciente) consiste que debe ser trabajada como un recuerdo olvidado o síntoma. Es así que el deber del terapeuta consiste en tratar de analizar estas fotografias inconscientes disociativas, ayudando al paciente a procesar el shock de manera explícita, así consiguiendo ordenar las circuitos témporo-espaciales primigenios.

Estas huellas mnémicas de la percepción han sido influidas por la cognición del paciente en cada esfera de su desarrollo psicosexual, posible núcleo de conflicto originario, que a lo largo de la vida, registrados y evocados a través de su prisma personal de fuerzas de hábito o costumbre, o condicionamientos inconscientes. Lo que se entiende por "memoria sensorial" no es solo una red motora instintiva sino también del sistema límbico-amígdala-hipotálamo-hipocampo, toda una red de convergencias del sistema nervioso autonómico, y que está elaborada por un conjunto de redes neuronales en otros subsistemas o sistemas del sistema nervioso central cognitivo, pensamiento y razonamiento.

El punto de vista real, es que lo que existen son memorias sensoriales, recuerdos de sí olvidados temporalmente, importantes para conducir la vida del adulto, rememorar y evocar es un vehículo que implica una memoria del inconsciente; a nivel subcelular ARN que trata de recordar asociativamente sus episodios de vida, cuando son traumáticos forma un núcleo de conflicto, causado por emociones negativas que se conectan con las emociones suscitadas, archivadas que se vuelven reactivas y automáticas por cada centro independiente que cumple funciones disociadas que no alertan riesgos de archivos mecánicos ordinarios de una loca imaginación del paciente ante los posibles peligros de que la vida lo lleve de las narices. (MONTERO D, 2018)

Hay un subtipo de memoria: La asociativa y la de habituación. Esta última archiva inconscientemente las circunstancias constantes que liberan nuestra atención, dirigiéndose a lo nuevo. En las neurociencias moleculares en psicología de las emociones, se dice que prevenir estos archivos es ser conscientes de sí, y tener una conciencia que se trata de la memoria automática de una proteína llamada Turbulina en los cito esqueletos protoplasmáticos de la célula neurona en el ARN dice lo que tiene que hacer el ADN. Es aquí donde las neurociencias moleculares en Psicología, proponen se forman en el llamado inconsciente subcelular ( en proteínas y enzimas), como subsistemas específicos que se 
encargan del recuerdo de imágenes en tanto el dato se forma en información pensamiento, o sea, probabilidades del electrón, y que la palabra cumple una función asociativa incluso de varias redes convergentes neuronales que especializan áreas en el recuerdo, ya sea de sustantivos o conjugación de verbos. (MONTERO D, 2018)

Así tenemos que los recuerdos emocionales se mantienen a largo plazo con firmeza porque estos datos movilizaron dichas emociones (negativas o positivas) y determinados sentimientos. Asi vemos sus correspondientes conexiones de la amígdala al córtex todos los razonamientos. Matemáticos en el lóbulo Parietal (LP), espaciales en el lóbulo Occipital (LO), verbales en el lóbulo Temporal (LT) y los más desarrollados, se llevan a cabo en el Lóbulo Frontal, donde se programa conductas que son mucho más lentas en asociaciones; que las asociaciones que van a la amígdala que son mucho más veloces, asi mismo las motoras instintivas tienen su propia lógica refleja por curso años desarrollo filogenético son muy antiguas y con esto, se constataría lo relativo de la fortaleza de lo irracional sobre lo racional de la base de datos del inconsciente (MONTERO D, Ana María 2018).

Asimismo, Feldman (1999), plantea que quizás se necesite en el futuro una neuroimagen y muchas tomografias (TAC-PET o SPECT) que revelen cambios significativos en el control cortical. A mi opinión es posible que las reacciones emocionales a veces equivoquen el pensamiento y vicever- sa, lo que si señalo que constatar y analizar esto es un método de observación objetiva, muy útil como criterio de éxito en el tratamiento mixto del psicoanálisis y las investigaciones que realizo en neurociencias moleculares en Psicología.

Continuando con esta línea de las neurociencias moleculares en psicología el inconsciente, se ha observa por huellas mnémicas en el hemisferio derecho, generadas como respuesta del SNA que pudo condicionarse en la niñez. Asimismo, también se ha evidenciado que en cuestión de detectar los elementos no verbales, el hemisferio derecho es el especialista, así como los paraverbales; como tono de voz, gestos. También es encargado de la vocalización humana, que es la prosodia, que le da el tono subjetivo al habla. Y con esto, podemos afirmar que cómo se dice una palabra, puede pesar más que el qué. Y para culminar esta idea, es bien sabido que los infantes pueden percatarse si la expresión verbal de sus padres tiene una connotación afectiva $u$ hostil, sin mencionar que el niño en esos primeros años de vida aún cuenta con un desarrollo cognitivo precario. De igual modo, un adulto cuando le hablan en un idioma diferente, es capaz de darse cuenta del tono emocional a pesar que no entiende el contenido. En suma, el hemisferio derecho da el contexto del mensaje y el izquierdo el texto. inconsciente (MONTERO D, 2018).

Galin (1974), refiere que "han sugerido que la racionalidad está asociada con la cognición lógica y analítica 
del hemisferio izquierdo", este dato se correlaciona con lo que Freud (1973), dice: "llamó pensamiento de proceso secundario; mientras que el modo de funcionamiento mental impresionista y no silogístico del hemisferio derecho sería propio de lo que en la nomenclatura freudiana se conoce como pensamiento de proceso primario".(obra de FREUD en sus tres tomos I, II y III)

E1 SNC está compuesto en hemisferio derecho (emocional) e izquierdo (racional), por tanto los archivos y zonas de registro de los conocimientos en ambos hemisferios devienen de investigaciones realizadas por los sesenta con Gazzaniga (1992), "en pacientes cuyo cuerpo calloso interhemisférico había sido seccionado quirúrgicamente como tratamiento de epilepsias refractarias a los fármacos anticomiciales de aquellos años. Normalmente, claro está, los dos hemisferios funcionan de forma conjunta, de tal modo que la percepción y la consciencia constituyen un todo continuo". Asimismo, otro ejemplo en un experimento de Gazzaniga (1992), en donde enseña la foto de un varón en paños menores al hemisferio derecho aislado de una mujer, narra: "y ella afirma que no ve nada, pero simultáneamente se rie nerviosamente y tiene respuestas autonómicas como aceleración del pulso o sonrojo". Paniagua (2000), refiere, al respecto, que "en efecto, cuando los datos de información se hallan secuestrados en el hemisferio derecho, el sujeto no es sólo incapaz de hablar de ellos, sino que, además, puede no ser consciente de poseerlos". Estos da- tos son de profunda relevancia para el psicoanálisis y el inconsciente.

\section{Los Mecanismos de Defensa como encubridores de los sintomas}

En efecto, La represión y el aislamiento del afecto son los mecanismos de defensa mejor estudiados en psicoanálisis. Si se presenta al hemisferio cerebral derecho de un paciente con split brain un mensaje visual como: "Vaya hacia la puerta”, el sujeto se levantará de su silla y se dirigirá a ella. Si se le pregunta entonces por qué se ha incorporado, dará respuestas (su hemisferio izquierdo) del tipo: "Voy al cuarto de baño" o: "Es que iba por un refresco". Al psiquismo humano le resulta dificil conformarse con simples "no sé" $y$, así, el hemisferio izquierdo, intérprete de las informaciones, intenta proveer de sentido y dar explicaciones causales a los estimulos que recibe, sean éstas auténticas razones o bien racionalizaciones, esto es, justificaciones defensivas destinadas a disminuir la ansiedad afectiva (Paniagua, 2000).

El investigador Wilder Penfield junto a su equipo de investigación en los cincuenta hicieron unas investigaciones respecto al mecanismo de defensa de la racionalización.

En su exploración con estimulación eléctrica de áreas del cerebro bajo anestesia local en 
pacientes que iban a requerir tratamiento quirúrgico para determinadas modalidades de epilepsia, encontraron que cuando, por ejemplo, se estimulaba una región motora $y$, por consiguiente, se producía la contracción de algún grupo muscular, éstos tendian (a veces tras una reacción de sorpresa inicial) a atribuir su movimiento involuntario $y$ sin propósito a algún tipo de motivación presentable (Penfield, 1954).

Y de la cita anteriormente mencionada, Rodríguez, J., se percató que en sus estudios de cerebros humanos implantados con electrodos, halló:

En uno de nuestros pacientes, la estimulación eléctrica de la parte anterior de la cápsula interna producía torsión de la cabeza con un desplazamiento lento del cuerpo hacia un lado en una secuencia de apariencia normal $y$ con un sensorio intacto, como si el paciente estuviese buscando algo...". Lo interesante de la experiencia es que el paciente consideraba espontánea esta actividad provocada y siempre la justificaba con una explicación razonable. Así, cuando se le preguntaba qué estaba haciendo, sus respuestas eran, 'estaba buscando mis zapatillas', 'escuché un ruido' (Rodriguez, 1969).

La moraleja que se deduce de las citas mencionadas es que las explicaciones y deducciones causales, actividad que compete al hemisferio izquier- do, pueden ser incorrectas, a pesar que el individuo tenga certeza de su veracidad. Esto es una característica de autoengaño del psiquismo humano que debemos manejar para no caer en el círculo vicioso.

Respecto a los fenómenos del proceso sensoperceptivo existen receptores de los sentidos (gusto, oído, olfato, visión, tacto, presión temperaturas) que son de Percepción inconsciente (Montero, 2016). Ahora en el siglo XXI existen muchas investigaciones en neurociencias moleculares cognitivasafectivas y otras con convergencia de redes que confirman el inconsciente luego de un tiempo incuantificable de desinterés e incredulidad en su existencia y en su rol que implica la percepción y la memoria. Al respecto, Sidis (1898) dice: "Hace más de un siglo que tenemos constancia experimental de la existencia de la percepción inconsciente". Posteriormente, en 1960, Pötzl, O., refiere "en una famosa serie de experimentos halló que un grupo de voluntarios expuestos a unos dibujos complejos durante una fracción de tiempo que hacía imposible su reconocimiento consciente (una décima de segundo), recuperaba en sus asociaciones y en sus sueños fragmentos de dichos dibujos". Ahora, mencionaré otra investigación experimental que hace énfasis en la base física del inconsciente.

En 1993, un neurofisiólogo, Libet, B., se percató que:

Describió cómo bastaba un impulso eléctrico de 10-20 milisegundos aplicado a la piel para 
producir una respuesta evocada electroencefalográficamente detectable en el córtex sensorial. Sin embargo, este estímulo no era percibido conscientemente por el sujeto hasta transcurrir 500 milisegundos. Al parecer, la respuesta evocada a los 10-20 milisegundos inicia una serie de reacciones corticales que culminan con la percepción consciente del estímulo, aunque el sujeto no se da nunca cuenta de dicho retraso; es decir, siempre tiene la experiencia subjetiva de simultaneidad (Libet, 1993).

Este fenómeno es de particular atención para los psicoanalistas que ya que demuestra como la toma de consciencia de este fenómeno tiene un retraso, a pesar que el individuo tenga la sensación subjetiva de instantaneidad del insight.

Posteriormente, Libet arguye;

Midió también este periodo de latencia y recogió las experiencias subjetivas de los individuos del experimento, hallando que, en efecto, la acción neuronal ha de persistir durante aproximadamente medio segundo (de preparación inconsciente) antes del movimiento muscular, que es percibido como simultáneo a la decisión voluntaria. Este momento preparatorio podría también ser suficiente para movilizar mecanismos psicológicos de defensa ante estímulos ansiógenos (Libet, 1993).

Wong (1999), al respecto, alega
"Hay ya, de hecho, considerables evidencias neurocientíficas de la existencia de señales inconscientes del miedo, de la angustia, que son las que, automáticamente, constituyen siempre el prólogo de las maniobras defensivas". El psicoanálisis mantiene la postura de que existen fenómenos mentales que son inconscientes por su contenido que genera ansiedad y culpa: Es por esto que la limitada consciencia de muchos estímulos externos e internos que afecta el ánimo del individuo no tienen una percepción consciente.

Frente a esta ansiedad surge probablemente el mecanismo de defensa de la represión, que se encarga de que la ansiedad permanezca en un estado inconsciente.

La psicología experimental apoya el mecanismo de defensa de represión como una caja tapada de recuerdos inconscientes, y para esto, Anderson et al. (2004), plantea que "identifica por medio de resonancia magnética funcional IRMN los sistemas neurales implicados en el proceso del olvido de los recuerdos indeseados". Para esto, profesionales de la salud mental aceptan que la mayoría del tiempo de la vida mental trabaja a nivel inconsciente, fenómeno corroborado por las técnicas de neuroimagen. Incluso, Freud habló de la idea de actividad psíquica inconsciente, diciendo del principio de economía de la energía donde se canaliza y expulsa el dato o la información con modificaciones (caso anormal) y $\sin$ modificaciones (caso normal). Este concepto había sido considerado un absurdo, hoy las neurociencias lo 
hacen evidentes al interactuar con el paciente sometido a una tomografia de imágenes de resonancia magnética nuclear IRMN donde se observa claramente la orientación del hidrogeno cuando la célula pierde energía o está enferma.

En mi rol de neurocientífica también afirmo que muchas escenas de nuestra vida son invisibles a la conciencia y reales en la inconsciencia, esto acaba generando mayor interés en ser buscadores de sí mismos y/o los investigadores que nos auto observamos y constatamos que existen fenómenos psíquicos inconscientes, $\mathrm{y}$ también preconscientes y de conciencia objetiva pero solo si al ser le sea posible integrar los centros y funciones psíquicas pensando lo que sentimos, sintiendo lo que pensamos y con un hacer fluido y equilibrado de centros, funciones redes, áreas de archivo de fenómeno que en sí serian la consciencia objetiva. La consciencia es una cualidad psíquica especial superior que supone una percepción de todo en cuanto existe en una a organización mental.

El área de la consciencia se estudia en base al método de autoobservación y constatación en sí mismo de hechos, de circunstancias de vida (experiencia vivida) que se puede enfocar-focalizar desde la atención activa, reflexiva lúcida, porque cada uno es una impresión pero al repetirse en simultáneo o paralelo a otras experiencias del foco consciente.

El conducir un carro sin atención mezcla ambas sensaciones: Atención y Consciencia mecánica u objetiva reflexiva son fenómenos de naturaleza psicológica de los centros cognitivo, afectivo, motor e instintivo ( a veces mecánicos otras veces centros superiores) que nos permite darnos cuenta de poder tener consciencia de nuestros procesos cognitivos y del hecho mismo de Ser Conscientes, que requiere del estudio de sí y el recuerdo de sí. Aún es poco sabido cuáles son los mecanismos fisiológicos automáticos ordinarios de nuestra maquina humana, cuerpo físico y no hacemos un espacio de silencio, de meditación sintiéndonos con atención activa anhelando que nada nos separe de nuestra esencia, tal vez la personalidad adquirida de un nivel sociocultural alto sea un buen bastón para dicha consciencia objetiva por adquirir, trascender hacia la energía más alta por la que fuimos creados en el polvo de estrellas que somos.

Se sabe, desde luego, que nuestro cerebro como fundamento físico (base material) tiene también energías sutiles en su funcionamiento cortical, sub cortical, medular, del cerebelo, hemisferio izquierdo, tálamo, hipotálamo metabólico nutricional del oxígeno, impresiones y de la ingesta de alimentos como substrato químico que estos alimentos conectan con circuitos de los sistemas neuromoduladores noradrenérgico (cólera) y adrenérgico (miedos), serotoninérgico (principio de placer) y colinérgico. Cabe observar también que es mucho lo que aún se desconoce sobre la neurobiología de las moléculas de las emociones (neu- 
ro péptidos) en la atención consciente versus inconsciente.

\section{E1 proyecto Genoma Humano y Cerebro Humano}

Al respecto, Paniagua (2004), alude: "El cerebro humano es el objeto conocido más complejo del universo. Aunque este órgano excepcional da cuenta de sólo el 2\% del peso corporal, aproximadamente la mitad del genoma humano está encargado de su codificación". El desarrollo cerebral consigue alguna forma de equilibrio entre la carga genética y la información adquirida, entre la programación inflexible y la adaptación funcional. Las investigaciones corroboran que las influencias ambientales pueden determinar la expresión de lo genético. Al respecto, Torsten Wiesel (1982), neurobiólogo de Harvard: Sostiene "Es posible que la sensibilidad del sistema nervioso a los efectos de la experiencia represente el mecanismo fundamental por el que el organismo se adapta al entorno durante su crecimiento". Aunque en el sistema nervioso central muchos circuitos siguen el dictado genético, el genoma de por sí no basta para determinar qué conexiones acabarán siendo las más funcionales. Las investigaciones en neurociencia de estos últimos años han mostrado que las interacciones con el entorno condicionan, hasta grados insospechados hace unas décadas, los patrones de secreción endocrina, las reacciones inmunológicas y la arquitectura microscópica de las redes nerviosas. Existe mayor plasticidad, por lo general, en la red neuronal de las zonas corticales prefrontales, donde pueden crecer nuevas dendritas y establecerse nuevas sinapsis virtualmente a lo largo de toda la vida. Esto es lo que nos permite adquirir información (ampliar nuestro vocabulario, aprender chistes, nuevos itinerarios, etc.) en edades avanzadas. Por otra parte, Papez (1937) menciona que "los circuitos límbicos subcorticales (los núcleos talámicos anteriores, la circunvolución cingular, hipocampo, complejo de la amígdala) que se establecen en la infancia temprana y que constituyen el cerebro generador de emociones primarias poseen una menor plasticidad funcional y tienden a ejercer efectos mucho más constantes en el desarrollo psicológico". Esta inflexibilidad podría parecernos escasamente adaptativa y, ciertamente, lo es en casos de aprendizajes o condicionamientos patológicos; sin embargo, es precisamente la mayor constancia de estos circuitos nerviosos la que da cuenta del fenómeno etiológico del imprinting o "troquelado" y, en el ser humano, es lo que explica el vínculo prolongado, tan peculiar de nuestra especie, del niño con su madre como fuente de sensaciones de placer y seguridad.

En la neurobiología molecular del cerebro y sus funciones energéticas sutiles psíquicas se sugiere una clara respuesta a este respecto: ambas teorías son ciertas.

El hecho de que estas últimas influencias puedan tener en ciertos periodos críticos consecuencias irre- 
versibles sobre la arquitectura de las moléculas de las emociones del punto de vista neurohistológico, que tiene para el psicoanalista y para las neurociencias moleculares en psicología una lectura clara de que, además de la patología por conflicto intrapsíquico (funcional) para la que fueron ideadas sus técnicas, existe lo que Anna Freud (1974) conceptuó como "psicopatología por déficit (estructural), no susceptible de un tratamiento psicoanalítico clásico, sino de otros abordajes técnicos de carácter paliativo".

Existen 96 leyes fisicas en la actividad nerviosa superior y base material del cerebro y sus energías psíquicas sutiles de los receptores sensitivos que cuentan con las convergencias actuales entre la neurociencia y el psicoanálisis. Es sabido que para el desarrollo ontogenético de las distintas categorias de la percepción, es necesario que el córtex sensorial reciba en un periodo crítico ciertos estímulos especificos.

Esto se ha estudiado sobre todo en el sistema visual, pero se considera un principio general aplicable a otras modalidades sensoriales.

En palabras de Wiesel (1982), uno de los principales investigadores del desarrollo funcional de la visión:

Los mecanismos innatos dotan al sistema visual de conexiones altamente especificas, pero es necesaria la experiencia visual temprana para el desarrollo completo del proceso. Los experimentos de privación sensorial muestran cómo dichas conexiones ner- viosas pueden ser alteradas por las influencias externas en periodos criticos, [...] pero bien pudiera ser que otros aspectos del funcionamiento cerebral, como el lenguaje, las tareas perceptuales complejas, el aprendizaje, la memoria y la personalidad tuviesen programas análogos de desarrollo (Wiesel, 1982).

El desarrollo intrauterino proporciona sólo la matriz topográfica de lo que van a ser las conexiones nerviosas en las áreas sensoriales. Basándose en una serie de estudios en animales y en humanos, Allan Schore (1994), de la Universidad de California, asegura que:

Existe tal periodo critico entre los seis y los doce meses para el desarrollo de circuitos en el córtex prefrontal, que regula la expresión de los estados afectivos. Para su desarrollo normal parece imprescindible que durante este periodo crítico el bebé tenga una interacción con la madre (o figura materna) que suponga un contacto visual y auditivo determinado (las sonrisas, las palabras amorosas) (Schore, 1994).

Aunque la influencia de los elementos ambientales sobre el sistema nervioso central se encuentra limitada por la dotación genética básica del individuo, también está comprobado que una estimulación sensorial precaria disminuye el grado de arborización dendrítica. Como han puesto de manifiesto Greenough et al. (1987), “el 
encéfalo de las ratas que en su jaula tienen laberintos y accesorios estimulantes de la atención y la actividad pesa considerablemente más que el de aquellas otras que sólo se mueven entre cuatro paredes". Los psicoanalistas han de interesarse en si la transferencia altera las redes neuronales encargadas de la patología neurótica, la corrección de estas.

Glen Gabbard (2000), de la Universidad Baylor de Houston, escribió: "Si consideramos la psicoterapia como una forma de aprendizaje, el proceso que se desarrolla ha de producir alteraciones en la expresión genética $\mathrm{y}$, por tanto, ha de ser capaz de modificar de algún modo las conexiones sinápticas".

La secuencia de un gen no puede ser cambiada por la influencia ambiental, pero sí su función transcriptora, es decir, la capacidad de fabricación de proteínas específicas. Los experimentos clásicos de Kandel (1976) con la Aplysia californica, un invertebrado marino hallaron que "el aprendizaje tenía como correlato físico una modificación permanente de las conexiones sinápticas entre las células. Esta modificación consistía no sólo en un aumento del número de sinapsis neuronales, sino también en un fortalecimiento de las ya existentes en términos de un incremento en el flujo de los transmisores químicos liberados por las terminales presinápticas".

\section{Las investigaciones sobre el cerebro de animales superiores}

Con más de una década de sucesivas investigaciones realizadas desde el 2003 al 2015, producto de las cuales la autora hoy ofrece a la comunidad científica esta obra con nuevas perspectivas donde convergen las neurociencias moleculares en psicología, proponiendo nuevas tendencias ante el avance de investigación y de la tecnología de punta de la nanotecnología.

En esta obra se abarca investigaciones desde los efectos de las bases biológicas moleculares, como los neurotransmisores, sus carencias, las diversas funciones de los minerales como el zinc, que también es una coenzima, su estudio abarca los cambios moleculares que están en relación con los aspectos comportamentales de los sujetos experimentales, efectos de estrés, depresión, efectos sobre el sueño, efectos en la gestación, en la memoria, en su interrelación con los aspectos afectivos y las preferencias psicosociales.

De esta forma: Se trata de tender puentes entre las variadas ciencias que incluyen las neurociencias; Física IV o Física Cuántica, Electromagnetismo, Ing. Informática, Medicina Humana, Neuropsicologia, Biología Molecular, Farmacología, Psicología Experimental en Psicogenética.

El conocimiento científico en Neurociencias incluye los niveles, genéticos, metabólicos, funcionales, inconscientes, conscientes, integración de centros cognitivo con el afectivo y 
motor instintivo, así como estudio de la senescencia o apoptosis celular. Conocer los efectos de estos niveles no sólo parte de hechos y la plasticidad neuronal sino trata también de la formulación de las posibles soluciones y es en base de la observación de las propias necesidades de la condición humana de conocer su máquina, anatomía y fisiología ya que el hombre ha generado instrumentos como: tomógrafos (TAC-IRMN, SPECT, MEG) todas máquinas de tecnología de punta, que van captando las transformaciones de los electrones (hidrógenos) en el curso y contenido de los fotones como fuentes de energía sutil psiquica del potencial de células normales o anormales déficit que revela a nivel sub-celular variaciones en frecuencia densidad y velocidad de la energía, como es evidente en la senescencia o apoptosis de la muerte celular por envejecimiento. En el caso de microscopios electrónicos, el estado celular convierte los acontecimientos comportamentales moleculares en diagnósticos globales sobre el estado de salud física o mental de los 35 órganos y 9 sistemas que posee el ser humano.

El conocimiento observado en las presentes investigaciones de neurociencias moleculares en Psicología da por establecido el arte objetivo que reflejan los fotones en el minimundo de la nanotecnología celular, lo cual permite que evolucionen las creencias científicas de la verdad del hombre confrontada con evidencias tecnológicas, tomógrafos u observaciones de microscopios electrónicos, como nue- vas estrategias que rompen los paradigmas, el uso de terapia comportamental o de terapias celulares, CM células madres, uso de Chips en la biónica, y así nacen soluciones descriptivas de un mundo interno molecular enteramente digitalizado en microscopios electrónicos de efecto de Túnel.

Finalmente, es la investigación acuciosa la que me hace buscadora científica en Neurociencias Moleculares en Psicología, siguiendo las huellas de Tolman, (Psicólogo Norteamericano) Kandel, Stone, Poppe y otros neurocientíficos que aspiran al encuentro de nuevos momentos de diagnóstico nuclear del ADN- ARN del comportamiento de las proteínas turbulinas en los citoesqueletos y descifrar los cálculos cuánticos que hace el ARN al llevar información al ADN y develar la verdad de los estados energéticos sutiles psíquicos biomoleculares en el interior de las células y por ende en las manifestaciones del cuerpo.

Los títulos de estas investigaciones en Neurociencias Moleculares en psicología son: Influencia del Glutamato Monosódico (MSG) en la Memoria de Animales Experimentales (Ratas Albinas) - 2003; Efectos de los Triptófanos en TCA - Anorexia- Bulimia (Animales Experimentales Ratas Albinas) - 2004; Efectos de la Aromaterapia Sobre las Citoquinas y el Estrés (en animales Experimentales ratas albinas comparativamente en humanos) - 2005, Efectos del Zinc sobre la Memoria Espacial y el Estrés (En animales experimentales ratas albinas) - 2006. Efectos de la Carencia de Sueño Asociados 
a un Rendimiento Motor y su Relación con el Sistema Inmune-Gastrointestinal (En animales experimentales mamíferos: Equinos [yeguas] comparativamente Humanos [jinetes] ) - 2007. Efectos de la Memoria Retrograda y Aprendizaje Negativo en el Estrés Post-traumático en muestras independientes (En Animales Experimentales: Yeguas -Jinetes) - 2008 al 2009; Efectos en el Comportamiento Gestante con Déficit Afectivo y Deterioros Orgánicos (Animales Experimentales: Gestantes Mujeres Comparativamente Yeguas) en el 2010; Efectos del Comportamiento Depresivo y el Deterioro Molecular Celular. (En Animales Experimentales en Muestras de Mamíferos: Equinos y Humanos) en el 2011; Efectos Neuropsicológicos de la Radiestesia en Muestras Independientes. (Mamíferos Experimentales en Yeguas Y Jinetes)- 2012, Efectos Comportamentales de la Resiliencia en el Poder Afectivo en grupo de Jinetes del Hipódromo (En Jinetes) en el 2013; Efectos de los Sentidos en la Memoria Sensorial: "El Recuerdo-Estrés y su Relación con los Hemogramas"(En Animales Experimentales Muestras De Mamíferos: Equinos-Humanos) en el 2014; Efectos de Interacción Animal y Humana en Comunicación y Comportamiento Molecular (Aplicado en Neurociencias Moleculares Aves y Mamíferos) en el 2015.

He podido observar que todas muestran las reglas de la vida: Paradoja porque la vida es compleja en los seres humanos, hay que mantener el humor cuando uno investiga, como una fuerza inconmensurable y que pueden existir cambios micro estructurales y macro estructurales cósmicos que redundan en la actividad nerviosa superior, en el tejido nervioso, a nivel celular de las neuronas y glias, en los neuropéptidos, en los neurotransmisores; estos son dependientes de la las impresiones y asociaciones que interaccionan con el entorno.

7. La corteza prefrontal retiene más que ninguna otra región cerebral las capacidades plásticas de su desarrollo temprano; especificamente el córtex órbito-frontal que continúa mostrando a lo largo de toda la vida las características histológicas y bioquimicas propias de su ontogenia.

Esto induce a pensar que los cambios producidos en tratamientos interactivos verbales pueden tener como substrato estructural esta peculiar plasticidad.

Pally (1977), señala que:

La región órbito-frontal es funcionalmente responsable de la capacidad de empatizar con los sentimientos de otros $y$, por tanto, se halla particularmente implicada en los comportamientos sociales. Antes del tercer mes, las fluctuaciones emocionales del bebé se encuentran mediadas por estructuras limbicas subcorticales, en especial por la amigdala. Después de esos meses iniciales, las primeras sonrisas del bebé anuncian el comienzo de la maduración del córtex 
órbito-frontal. La consiguiente interacción entre la madre y el niño seguramente estimula la mielinización de los circuitos nerviosos que conectan el córtex sensorial con el órbitofrontal (Pally, 1977).

Un estudio publicado por Schwartz y cols. (1996), que ha tenido gran resonancia, ha sido el llevado a cabo

En pacientes antes y después de un tratamiento psicológico terminado con éxito, siguiendo la evolución de tomografías cerebrales de emisión de positrones (PET), que miden el metabolismo de la glucosa en el sistema nervioso $y$, por tanto, el nivel de actividad neuronal. En este estudio se halló que se producían cambios significativos en la actividad metabólica de la corteza órbito-frontal y sus conexiones subcorticales (Schwartz y cols, 1996).

En otro estudio similar publicado por Viinamäki y cols. (1998), mostraron "cómo un paciente deprimido sin farmacoterapia alguna, con solo un año de psicoterapia dinámica de una sesión semanal, pasó de una captación de monoaminas notablemente reducida en el área prefrontal medial y el tálamo a una captación normal, comparada con controles".

\section{Los circuitos limbicos subcorti- cales}

Las redes neuronales por convergencia pueden en neurociencias moleculares en Psicología y en tratamiento de psicoterapia; ser desactivadas o inducidas, relativo a la corriente eléctrica $\mathrm{mV}$ (mini voltios) y Neuropéptidos moléculas químicas vehículos de transmisión de información, producen un circuito o red neuronal que actúa por un campo magnético (Spin del Hidrogeno -Electrón). El Circuito giratorio del Spin $\mathrm{H} 1$ es acoplado al inductor CPU y es capaz de generar una corriente eléctrica, que permite ver el estado energético de la neurona o célula en estudio en una gama de Fotones o colores:

- FOTONES : Donde Azul oscuro = indica célula en proceso apoptosis o muerte celular; verde $=$ deterioro moderado de la energía de la célula; Naranja= leve perdida; Rojo = Normal $\mathrm{mV}$ (mini voltios) de la estructura estudiada.

- $\quad$ El pC/PCC sería el único nodo de la RND desactivación que paradójicamente interactúa, virtualmente, con todos los otros nodos, mediando posiblemente su actividad.

- La fuerte interconectividad entre el pC/PCC el RND y el resto de las redes aportaría la hipótesis de que esta región actuaría como un nodo de convergencia o red de convergencia donde la información procesada en los dos subsistemas de esta red es integrada, e informa sobremapeo.

Existe en los cerebros de los investigadores y científicos una inflexibilidad al asumir el rayo de la creación donde el hombre es parte del pensamiento de Dios, lo cual podría parecernos escasamente adaptativa esta frase real cuando aceptamos el Budismo, el 
cristianismo de los esenios o los evangelios. Lo cierto es que nuestras instrucciones cerebrales pocas veces son trascendentes y justas con la creación ciertamente, todo en la vida es hábito de aprendizajes instrucciones recibidas genéticamente, por influencia del entorno, la gran naturaleza nos da aprendizajes o condicionamientos normales; otras veces se convierten patológicos porque el inconsciente los tomó y los ocultó, y nunca los hemos hecho objetivamente conscientes.

\section{Investigaciones sobre el cerebro de animales superiores}

Estas investigaciones muestran sin duda alguna, pueden existir cambios micro estructurales, tanto en el tejido nervioso, célula, subcélula núcleo ARN y ADN donde las vibraciones de los spin son probabilidades de hidrógenos (electrones) en el cortex, subcortex, médula espinal y cerebelo dando lugar al pensamiento, afectos, motor e instintivo del Ser, lo que denomino en este artículo huellas sin tiempo del inconsciente condicionadas por la fuerza de hábitos o costumbres y dadas en la educación que pasó por las instituciones con dependencia de la interacción con el entorno.

La corteza prefrontal retiene más que ninguna otra región cerebral las capacidades plásticas de su desarrollo temprano; específicamente el córtex órbito-frontal que continúa mostrando a lo largo de toda la vida las características histológicas y bioquímicas propias de su ontogenia.

\section{Los sueños}

En relación a este punto se tiene los trabajos de J. Allan Hobson, neurofisiólogo y profesor de Psiquiatría de Harvard, el cual es conocido especialmente por sus investigaciones, en los años setenta y ochenta, sobre los procesos fisiológicos generadores de la actividad onírica (el sueño REM). Hobson (1994) halló que "ésta se iniciaba por la descarga neuronal de ciertos núcleos pontinos del tallo cerebral". Las proyecciones nerviosas de esta región mesencefálica estimulan otras regiones subcorticales y corticales, con la acetilcolina como neurotransmisor activador (en el estado vigilia que son los sistemas noradrenérgico y serotoninérgico, y los activadores de estas regiones). Se ha demostrado que el patrón de conducción de estas proyecciones es individual para cada cerebro. Estudios con PET demuestran cómo en el sueño REM aumenta la actividad casi de forma paroxística en el córtex sensorial y en el área límbica (asiento de las emociones primarias y la memoria), mientras que se reduce simultáneamente la actividad en las zonas prefrontales ("ejecutivas"). La inhibición del sistema prefrontal, responsable del pensamiento racional y el control de los impulsos, hace que éstos, junto con las vivencias emocionales y algunos recuerdos olvidados, puedan acceder a la consciencia. A todo esto puede vérsele una dimensión adaptativa, puesto que los sueños, esas asociaciones mentales de apariencia caprichosa, generados ante la relajación 
del funcionamiento prefrontal, permiten a veces llegar a formulaciones "impensables" en el estado vigilia. Se sabe además que la privación experimental de sueño REM empeora el rendimiento en aquellas tareas que requieren pensamiento creativo $\mathrm{y}$, por otra parte, que los pacientes en situaciones de estrés muestran un aumento de la actividad REM que puede contribuir al hallazgo de soluciones originales.

Es importante reseñar que, según Hobson (1994),

Aunque las lesiones en el puente de Varolio pueden hacer que se suprima el sueño REM, ello no impide que el paciente relate sueños. Por el contrario, cuando el puente está intacto pero se encuentra dañado el córtex prefrontal, se produce sueño REM, pero el sujeto no puede referir sueño alguno. Esto podría considerarse como evidencia de que la actividad onírica está provista de significados relacionados con la cognición y las emociones, y no es simplemente un "detritus neurofisiológico”, como estimó Hobson (1994).

De sus descubrimientos sobre el origen y secuencia fisiológicos del sueño REM, Hobson y McCarley (1977) habian llegado, en efecto, a la conclusión de que "los sueños carecían de sentido psicológico alguno". Según estos investigadores, sus hallazgos confirmaban que no existía otra perspectiva posible en la "comprensión de los fenómenos oníricos anatomofisiológicos".
Ciertamente, sus descubrimientos arrojan dudas sobre algunos aspectos de la psicogénesis y la elaboración de los sueños, tal como las concibió Freud hace un siglo, pero ¿niegan la existencia de una psicodinámica? Recuerdo haber escuchado decir al renombrado psiquiatra e investigador Seymour Kety (1972): "Llegaremos a conocer la fisiología e histología del sueño, pero no de los sueños".

Los sueños de todos los humanos están mediados por un mismo neurotransmisor (la acetilcolina), pero ¿qué nos dice eso de nuestras diferencias individuales y del hecho de que todos los sueños en una misma persona sean distintos?

Siguiendo esta manera reduccionista de razonar habriamos de pensar también que para comprender la psicosexualidad humana deberiamos prescindir de explorar científicamente las fantasías individuales al respecto y limitarnos a estudiar fenómenos como la inervación genital o las secreciones endocrinas correspondientes.

\section{Mente y su fisiologia sutil ener- gética cósmica}

Los fenómenos mentales no son asunto para decidir si el psiquismo posee o no base fisiológica. Naturalmente que la tiene. La intencionalidad, las motivaciones, los significados y cualquier otra manifestación psíquica normal o patológica, se hallan implementados neurofisiológicamente.

Ya en tiempos de Rudolf Virchow solía decirse que el cerebro segregaba 
pensamientos como el hígado bilis.

La mente es un epifenómeno del funcionamiento cerebral: una "propiedad emergente", como dicen los filósofos de la ciencia.

Se define una propiedad emergente como aquella conectada causalmente con elementos o fenómenos anteriores, pero que no constituye una cualidad de ninguno de ellos ni resulta de su simple adición.

La actividad psíquica es consecuencia de la descarga de grupos neuronales de determinadas áreas del cerebro, pero la mente no equivale sencillamente al funcionamiento de estas áreas cerebrales.

Las secuencias causa-efecto no son sinónimas de identidad. El trueno sigue al relámpago, pero el trueno no es el relámpago. La comprensión del sentido de la conducta y de las experiencias subjetivas se halla a un nivel de coherencia diferente de cualquier formulación de leyes fisiológicas. George Klein (1976), investigador y psicoanalista de la Fundación Menninger, sostuvo:

Puesto que las intenciones son personales y relacionales, los modelos adecuados para la búsqueda de fines no pueden ser formulados en términos de proposiciones impersonales [...]. Realmente, la lectura de las intenciones o coherencias en las acciones y experiencias de otros no puede hacerse en los acontecimientos fisiológicos, al menos no más de lo que la finalidad del transporte puede ser corregida a partir del funcionamiento mecánico del automóvil (Klein, 1976).

Michael Polanyi, químico, conocido filósofo de la ciencia y profesor de la Universidad de Manchester, escribió elocuentemente sobre la necesidad de incluir las experiencias y estados subjetivos dentro del campo del escrutinio científico.

Polanyi (1965), señaló: "Un neurofísico que observase los procesos que tienen lugar en la retina y en el cerebro de un hombre que está mirando algo, no sería nunca capaz de interpretar, a partir de los acontecimientos físicos bajo estudio qué está viendo el hombre en ese algo". El estudio de la percepción, la memoria y la consciencia, en efecto, será siempre incompleto si no se añade al punto de vista objetivo al subjetivo.

\section{Conclusiones de mis investiga- ciones en neurociencias molecula- res en psicologia}

$1^{\circ}$ La mente recibe alimentos de manera inconsciente del aire en la respiración; en las dietas, las comidas y sus nutrientes; y en los receptores sensoriales en calidad de impresiones y asociaciones que son recuerdos olvidados, huellas sin tiempo, archivados en nosotros mismos.

$2^{\circ}$ Los efectos comportamentales debidos a la administración en exceso de ingesta de triptófanos en animales experimentales, ratas, y medir a través de las pruebas de laboratorio (análisis de sangre) y pruebas psicológicas comportamentales (la observación y 
registros de conducta en situaciones con y sin estrés) del desempeño animal; así mismo, evaluar episodios de vida alimenticia y encontrar la existencia o no de un patrón alimenticio codificador de la ingesta que puede causar efectos supresores del apetito.

Los triptófanos son aminoácidos que son obtenidos de la comida de carbohidratos (especialmente pan blanco) y que el cerebro asimila enervando ansiedad obsesiva, probablemente causando exceso de neurotransmisores que generan la activación del receptor 55H2A o supresor del apetito.

$3^{\circ}$ Los procesos sensoperceptivos que existen son un complejo y apasionante tema, como es la relación entre la aromaterapia y el estrés buscando sus bases científicas en Neurociencia. Existen datos que demuestran que todos los mamíferos, en los que se incluye el hombre y las ratas albinas, presentan interés en su esquema corporal y, por una situación relajante ante la aromaterapia del extracto de lavanda, dadas las propiedades químicas del mismo, los sujetos experimentales mostraron una respuesta fisiológica controlada por el bulbo olfativo y su interacción neuropéptica con el hipotálamo en condiciones experimentales con estrés y sin él; observándose por el tiempo de reacción empleado, la respuesta de dar la orden de relajación a sus contracciones musculares lisas y estriadas. Segundo, con respecto a la relación SNC y Sistema Inmune con respecto a los glóbulos blancos (monocitos, citoquinas - Interluquinas), el número promedio nor- mal es de $100 \mathrm{~cm} 3$. En la condición experimental "A" con ratas albinas con estrés se pudo observar que el tiempo de reacción promedio fue de $5^{\circ} .40^{\prime \prime}$ (cinco minutos / cuarenta segundos), con presencia de citoquinas proinflamatorias IL-1, IL-6 en un recuento promedio de $171.60>100 / \mathrm{cm} 3$. Sin embargo, en la condición B sin estrés, el tiempo de reacción promedio fue menor 3.20" (tres minutos / veinte segundos con presencia de citoquinas antiinflamatorias IL-4, IL-10 $\leq 100$ cm3. En la tercera, se observó con respecto al comportamiento humano que existió una gran diferencia entre las proporciones del tiempo de reacción entre los sujetos que se relajaron en las dos condiciones (con y sin estrés), logrando demostrarse que la Aromaterapia sí ayuda a reducirlas tensiones induciendo a un estado de relajación muscular.

$4^{\circ}$ La ingesta de zinc con vitamina $\mathrm{C}$ favorece el proceso cognitivo de la memoria espacial. Se pudo observar como los efectos peroxinitrito (estrés oxidativo) en mediadores de la fisiopatología de tejidos meníngeos y células hipotalámicas post mortem habian incrementado el número de glias por neuronas en los cerebros de las ratas albinas.

$5^{\circ}$ La fisiología del sueño trata de conectar los efectos comportamentales de la carencia de sueño en parámetros fisiológicos relacionados con el rendimiento motor, el estado del sistema inmune y gastrointestinal en yeguas y jinetes, ambos mamíferos participantes en carreras, quienes fueron 
sometidos a condiciones experimentales con y sin estrés.

$6^{\circ}$ Existen aprendizajes inconscientes negativos donde el estrés post traumático refleja desesperanza en las personas que han sufrido una experiencia negativa recordada retrógradamente respecto a carreras perdidas por fracturas, fisuras y golpes moderadamente impactantes en sus vidas profesionales.

$7^{\circ}$ En caso de los efectos comportamentales en gestantes con déficit afectivo y deterioros orgánicos, comparativamente entre mujeres y yeguas, la investigación se centra en la evaluación y análisis de los cambios emocionales y fisiológicos que se producen en el psiquismo de una gestante, evaluando estados ansiosos, niveles de depresión y estrés, correlacionados con un deterioro físico orgánico. El Déficit Afectivo presenta una correlación alta y significativa con Deterioro Orgánico en gestantes; por lo tanto una adecuada ingesta y estabilidad emocional permiten un embarazo sin mayores complicaciones.

$8^{\circ}$ Existen las moléculas de las emociones negativas con conexión entre los efectos comportamentales depresivos y deterioros orgánicos a nivel de moléculas de las emociones.

$9^{\circ}$ En las personas, existe una correlación alta positiva entre el comportamiento depresivo y el deterioro molecular. Por consiguiente, a mayor comportamiento depresivo, mayor deterioro molecular o viceversa. Esto quiere decir que a nivel molecular, las emociones negativas tienen su meca- nismo fisiológico electromagnético a nivel de canales iónicos de membranas celulares.

$10^{\circ}$ Cuando se investiga los efectos neuropsicológicos de la radioestesia en muestras independientes de mamíferos, como yeguas y jinetes, colocándoles un microchip de registro del comportamiento fisiológico de su cuerpo físico, lo investigado fue exitoso en la muestra de 30 sujetos participantes. No presentaron evidencia científica que los microchips implantados causaran alteración neuropsicológica alguna. El uso de microchips en caballos y jinetes no causó alteraciones neuropsicológicas o moleculares, ni variabilidad clínica en la agudeza visual o auditiva del usuario, incluyéndose ausencia de dolores de cabeza, mareos o pérdida de memoria

$11^{\circ}$ Es importante observar que el impacto de la resiliencia en el comportamiento afectivo, dado que la resiliencia es la capacidad que tiene una persona para superar circunstancias traumáticas como la muerte de un ser querido, un accidente, es como un sufrimiento inconsciente.

$12^{\circ}$ La memoria sensorial y los efectos traumáticos en la persona muestran una conexión entre los efectos comportamentales entre sentidos y la memoria sensorial, haciendo posible la observación sobre los posibles deterioros moleculares vía hematológica: VSG velocidad de segmentación elevada tal vez por efectos de recuerdos estresantes y circunstancias actuales de los sujetos estudiados, dadas medidas correlacionales de inestabilidad 
emocional y recuerdos traumáticos. A través de los hemogramas se puede tener una idea de que si se cumple con patrones estándares o hay diferencias significativas en cuanto carreras de resistencia y de fondo.

$13^{\circ}$ Los efectos de interacción animal y humana en comunicación y comportamiento molecular, observando cómo se presentan la conexiones entre la variable dependiente emisión de sonidos (trino o canto de ave, relincho de equinos), protestas (de jinetes), cuando están sometidos como variable de control a privación de ingesta de comida por 24 horas, efectos que se correlacionaron con estado de ánimo emocional de los sujetos participantes (aves, yeguas y jinetes ) con la variable independiente neuropéptidos en disminución o aumento de proteáceas (neuro péptidos) a nivel molecular de células ganglionares y del hipocampo observadas via hematológica.

Todos estos comportamientos son interactivos en la comunicación, haciendo posible la observación experimental descriptiva del comportamiento sonoro auditivo de aves, mamíferos (equinos y jinetes) que presentan patrones de impresiones auditivas sensomotoras (orgánicas) como reflejos de sus estados de ánimo con respuestas moleculares proteicas medidas hematológicamente, y conductuales socializantes de condicionamientos.

$14^{\circ}$ En los efectos de las manifestaciones del estado emocional y la sexuación humana en el comportamiento molecular del tercer núcleo intersticial (INAH-3) del Hipotálamo anterior, se demostró una relación significativa entre el estado emocional estable o inestable, las dimensiones de la estructura hipotalámica del tercer núcleo Intersticial anterior (INAH-3), frente al recuento hematológico de sus glóbulos blancos.

$15^{\circ}$ En esta última investigación, 2017, he podido observar que al ser diagnosticada la enfermedad de Alzheimer temprana (EA) con un grupo de ratas sanas, especie Rattus norvegicus (variedad albina); estas, como grupo de control (SC), se les daba diferentes tareas de memoria sensorial MS no verbal, en cuanto al recuerdo de posición y reconocimiento de dibujos abstractos. Todos los participantes completaron un protocolo integral para el diagnóstico de la demencia.

Sin embargo, al comparar con el grupo $\mathrm{SC}(\mathrm{n}=15)$ en un test estandarizado de aprendizaje viso espacial, los sujetos experimentales alimentados con 2,5 mg de erythrina edulis o Balu con EA rindieron significativamente más alto en MS (memoria sensorial) o MNV en comparación con el grupo SC, pero diferencias significativas entre EA en el recuerdo de posición. Asimismo, el grupo de EA mostró una tasa de olvido menor en el recuerdo de posición o reconocimiento de dibujos en comparación con los grupos SC. Estos resultados son interpretados en función de recuperación de los procesos neurocognitivos que explican el deterioro de la MS, memoria sensorial no verbal en la EA. 


\section{En resumen final}

Queria compartir que las investigaciones en Neurociencia Moleculares en Psicología demuestran que existe un comportamiento humano del estado emocional inconsciente con expresión molecular fotónica.

La fotónica es la ciencia de la generación, control y detección de fotones que tienen posibilidades asociativas de electrones con propiedades electromagnéticas estudiados en la física cuántica. Los dispositivos fotónicos de semiconductores incluyen:

-Almacenamiento del dato óptico, térmico, en donde la proteína Turbulina y los citoesqueletos del ARN dicen qué hacer al ADN inconscientemente.

- Las redes y circuitos funcionan fotónicamente como una fibra óptica en cada nervio de neurona a neurona, y asî lo demuestran los SPECT (tomografias computarizadas con un solo Fotón) con una alta tecnología actual.

- Impresiones en el cerebro inconsciente son como rayos láser de alta potencia en la subcélula a nivel de membranas externas a la célula y del núcleo.

- Las aplicaciones electromagnéticas de la Química Molecular tienen una expresión física de energía sutil (Datos e información) del inconsciente, donde la Medicina Humana puede elaborar microplaquetas (chips) en medidas Nanométricas, y en donde las comunicaciones de correlación de redes neuronales y circuitos producen y obtienen energía sutil por fusión térmica de los hidrógenos (Electrones); siendo los pensamientos, afectos, actos motores y sensitivos, probabilidades de electrones, lo que despierta gran interés científico y tecnológico en los últimos años por deducir las velocidades, densidades y frecuencias de mayor o menor voltaje electromagnético. Esto hace que hoy por hoy se haya delineado un área de investigación de indole Neurocientifico Molecular Nanométrico, con el objetivo de hallar la importancia de la posible causa psicológica inconsciente de todas las impresiones sensoriales y percepciones. Por ejemplo, las impresiones auditivas recordadas en el silencio influyen sobre la percepción sensorial humana y animal que causan una serie de vibraciones en las redes neuronales asociativas por convergencias simples y complejas en el córtex (diversos lóbulos $\mathrm{y}$ funciones de razonamientos), sub córtex cerebro emocional (SLD) donde tanto en las estructuras Hipotalámicas; Talámicas, la Amígdala como el Hipocampo (Centro de la Memoria), coordinan con la locus cereleus (centro de Atención) FRA (formación Reticular ascendente) que coordina la motricidad fina y gruesa y las probabilidades de movimiento vibratorio de electrones con respecto a las bases nitrogenadas del ARN del citoplasma y del ADN en los núcleos de cada neurona de las estructuras señaladas.

El hombre, como sintesis de todos los seres, tiene positivos funcionales de la Naturaleza, dado que es una semilla Universal de ella, y del cosmos, ya que somos polvo de estrellas, una sintesis de luz, sonido, formas de 
minerales, metales, agua, plantas, y animales con proporciones energéticas (psíquicas) y materiales (cerebro) e ignoramos la necesidad de vida interior que se manifiestan como individualidad de todas las experiencias vibratorias vividas en instancias de personalidad, llámese Ello o principio de placer (instintos de vida o muerte), Yo (principio de realidad), Yoes falsos, fantasias, locas imaginaciones o delirios seudoperceptivos), con un Superyo (Normas y valores) que esclavizan al individuo a su entorno externo-interno a una real posibilidad de integrar sus centros y funciones que expresen una conducta integrada (cognitiva, afectiva, motora e instintiva cohe- rente), siendo esta una transposición energética sutil de la creación y de la función universal cósmica, un testimonio de vida y sabiduría de la conciencia universal o, lamentablemente, de la ignorancia profunda sobre el cuerpo físico máquina-humana dentro de los siglos de sucesión de la esencia de nuestros hidrógenos que vibran haciendo al hombre inmortal y que gracias al trabajo consciente y sufrimiento voluntario en la vida, no se pierde en la esclavitud de lo ordinario, como si no tuviera instinto de conservación y seguridad personal, pues ignoran el deseo de conocer la necesidad del vivir armónicamente la vida y trascender a ella (Montero, 2017).

\section{REFERENCIAS BIBLIOGRÁFICAS}

Anderson, M. C., Ochsner, K. N., Kuhl, B, Cooper, J., Robertson, E., Gabrieli, S. W., et al (2004). Neural systems underlying the suppression of unwanted memories. Science. 303: 232-235.

Andreasen, P. J., O’Leary, D. S., Cizadlo, T., Arndt, S., Rezai, K., Watkins, G. L., et al. (1995). Remembering the past: Two facets of episodic memory explored with positron emission tomography. Am J Psychiat. 152: 1576-1585.

Damasio, A. (2001). El error de Descartes. Madrid: Critica.

Dawson, G. \& Fischer, K. W. (1994). Human Behavior and the Developing Brain. Nueva York: Guilford.

Feldman, A. (1999). MEM Neurosciences. http:/ /www.apsa.org, 8 de noviembre. Freud, A. (1974). A psychoanalytic view of developmental psychopathology. $J$ Philadelphia Assn Psychoanal. 1: 7-17.

Freud, S. (1973a). Introducción al narcisismo. Madrid: Biblioteca Nueva.

Freud, S. (1973b). La interpretación de los sueños. Madrid: Biblioteca Nueva.

Freud, S. (1973c). Lo inconsciente. Madrid: Biblioteca Nueva.

Freud, S. (1973d). Más allá del principio del placer. Madrid: Biblioteca Nueva.

Freud, S. (1973e). Proyecto de una psicología para neurólogos. Madrid: Biblioteca

Nueva. 
Gabbard, G. O. (2000). A neurobiologically informed perspective on psychotherapy. British J Psychiatry. 177: 117-122.

Galin, D. (1974). Lateral specialization and psychiatric issues: Speculation on development and the evolution of consciousness. Ann N Y Acad Sci. 299: 397-411.

Gazzaniga, M. S. (1992). Nature's Mind. Nueva York: Basic Books.

Greenough, W. T., (1987). Black JE y Wallace CS. Experience and brain development. Child Development. 58: 539-559.

Hobson, J. A. \& McCarley, R. W. (1977). The brain as a dream state generator: An activation- synthesis hypothesis of the dream process. Amer J Psychiat. 134: $1335-1348$.

Hobson, J. A. (1994). The Chemistry of Conscious States. Boston: Little, Brown. Joseph, R. (1996). Neuropsychiatry, Neuropsychology and Clinical Neuroscience. Baltimore: Wilkins \& Wilkins.

Kandel, E. R. (1976). Cellular Basis of Behavior: An Introduction to Behavioral Neurobiology. San Francisco: W. H. Freeman.

Kandel, E. R. (1983). From metapsychology to molecular neurobiology: Explorations into the nature of anxiety. Amer J Psychiat. 140: 1277-1293.

Klein, G. (1976). Psychoanalytic Theory: An Exploration of Essentials. Nueva York: Int. Univ. Press.

Libet, B. (1993). The neural time factor in conscious and unconscious events. En Experimental and Theoretical Studies of Consciousness. Nueva York: Wiley.

Montero, A. M. (2016). Investigaciones en Neurociencias Moleculares en Psicologia. Lima: Business Support Aneth.

Pally, R. (1997a). How brain development is shaped by genetic and environmental factors. Int J Psychoanal. 78: 587-593.

Pally, R. (1997b). How the brain actively constructs perceptions. Int J Psychoanal. 79: 1021-1030.

Paniagua, C. (2000a). Consciencia, cientificismo y multideterminismo en psicoanálisis. Rev Asoc Esp Neuropsiq. 76: 67-78.

Paniagua, C. (2000b). Neurobiología y psicoanálisis. Actas Esp Psiquiatr. 28: 53-59.

Paniagua, C. (2000c). Neurociencia y psicoanálisis. Archivos Psiquiatr. 63: 339350.

Paniagua, C. (2004). Convergencias actuales entre la neurociencia y el psicoanálisis. Ars Medica. Revista de Humanidades. 2:194-211.

Papez, J.W. (1937). A proposed mechanism of emotion. Arch Neurol Psychiat; 38: $725-735$.

Penfield, W. \& Jasper, H. (1954). Epilepsy and the Functional Anatomy of the Brain. Boston: Little, Brown. 
Piaget, J. (1950). La Construction du Réel Chez l'Enfant. Neuchâtel: Delachaux, Niestle.

Polanyi, M. (1965). The structure of consciousness. Brain, 88: 799-810.

Pötzl, O. (1960). The relationships between experimentally induced dream images and indirect vision. En: Preconscious Stimulation in Dreams, Associations, and Images. Monogr. 7. Psychol Issues. Nueva York: Int Univ Press.

Reise, M. F., (1984). Mind, Brain, Body: Toward a Convergence of Psychoanalysis and Neurobiology. Nueva York: Basic Books.

Rodríguez, J. M. (1969). Physical Control of the Mind. Nueva York: Harper \& Row.

Schore, A. N. (1994). Affect Regulation and the Origin of the Self. Hillsdale: L. Erlbaum.

Schwartz, J. M. et al. (1996). Systematic cerebral glucose metabolic rate changes after successful behavior modification treatment of obsessive-compulsive disorder. Arch Gen Psychiat. 53: 109-113.

Sidis, B. (1898). The Psychology of Suggestion. Nueva York: Appleton.

Tulving, E. \& Thomson, D. (1973). Encoding Specificity and Retrieval Processes in Episodic Memory. Psichological Review. 80(5), 353-373.

Viinamäki, H. et al. (1998). Change in monoamine transporter density related to clinical recovery: A case control study. Nordic J Psychiat. 52: 39-44.

Wiesel, T. N. (1982). Postnatal development of the visual cortex and the influence of environment. Nature. 299: 583-591.

Wong, P. S. (1999). Anxiety, signal anxiety, and unconscious anticipation: Neuroscientific evidence for an unconscious signal function in humans. $J$ Amer Psychoanal Assn. 47: 817-841. 